OPEN ACCESS

Edited by:

Jun Ren,

University of Washington,

United States

Reviewed by:

Ying Tan,

Southern Medical University, China

Ne Natalie Wu,

Fudan University, China

*Correspondence:

Junmeng Zheng

zhengjunmeng@126.com

Shi Liang

md02/s@mail2.sysu.edu.cn

${ }^{\dagger}$ These authors have contributed equally to this work

Specialty section:

This article was submitted to

Integrative Physiology,

a section of the journal

Frontiers in Physiology

Received: 19 March 2020 Accepted: 30 April 2020

Published: 05 June 2020

Citation:

Tao J, Wang Y, Li L, Zheng J and Liang S (2020) Critical Roles

of ELVOL4 and IL-33

in the Progression of Obesity-Related Cardiomyopathy via Integrated

Bioinformatics Analysis.

Front. Physiol. 11:542.

doi: 10.3389/fphys.2020.00542

\section{Critical Roles of ELVOL4 and IL-33 in the Progression of Obesity-Related Cardiomyopathy via Integrated Bioinformatics Analysis}

\author{
Jun Tao ${ }^{1 \dagger}$, Yajing Wang ${ }^{2 t}$, Ling $\mathrm{Li}^{1}$, Junmeng Zheng ${ }^{1 *}$ and Shi Liang ${ }^{1 *}$ \\ ${ }^{1}$ Department of Cardiovascular Surgery, Sun Yat-sen Memorial Hospital, Sun Yat-sen University, Guangzhou, China, \\ ${ }^{2}$ Department of Otorhinolaryngology, Sun Yat-sen Memorial Hospital, Sun Yat-sen University, Guangzhou, China
}

The molecular mechanisms underlying obesity-related cardiomyopathy (ORCM) progression involve multiple signaling pathways, and the pharmacological treatment for ORCM is still limited. Thus, it is necessary to explore new targets and develop novel therapies. Microarray analysis for gene expression profiles using different bioinformatics tools has been an effective strategy for identifying novel targets for various diseases. In this study, we aimed to explore the potential genes related to ORCM using the integrated bioinformatics analysis. The GSE18897 (whole blood expression profiling of obese diet-sensitive, obese diet-resistant, and lean human subjects) and GSE47022 (regular weight C57BL/6 and diet-induced obese C57BL/6 mice) were used for bioinformatics analysis. Weighted gene co-expression network analysis (WGCNA) of GSE18897 was employed to investigate gene modules that were strongly correlated with clinical phenotypes. Gene Ontology (GO) functional enrichment analysis and Kyoto Encyclopedia of Genes and Genomes (KEGG) pathway analysis were performed on the co-expression genes. The expression levels of the hub genes were validated in the clinical samples. Yellow co-expression module of WGCNA in GSE18897 was found to be significantly related to the caloric restriction treatment. In addition, GO functional enrichment analysis and KEGG pathway analysis were performed on the coexpression genes in yellow co-expression module, which showed an association with oxygen transport and the porphyrins pathway. Overlap analysis of yellow co-expression module genes from GSE18897 andGSE47022 revealed six upregulated genes, and further experimental validation results showed that elongation of very-long-chain fatty acids protein 4 (ELOVL4), matrix metalloproteinase-8 (MMP-8), and interleukin-33 (IL33) were upregulated in the peripheral blood from patients with ORCM compared to that in the controls. The bioinformatics analysis revealed that ELOVL4 expression levels are positively correlated with that of IL-33. Collectively, using WGCNA in combination with integrated bioinformatics analysis, the hub genes of ELVOL4 and IL-33 might serve as potential biomarkers for diagnosis and/or therapeutic targets for ORCM. The detailed roles of ELVOL4 and IL-33 in the pathophysiology of ORCM still require further investigation.

Keywords: ORCM, WGCNA, yellow co-expression module, ELOVL4, IL-33 


\section{INTRODUCTION}

The number of overweight and obese individuals has dramatically increased. In China, more than $10 \%$ of the adult population is obese (He et al., 2017; Qasim et al., 2018; Wang and Ren, 2018). While in America, the prevalence of obesity among adults is about 39.6\% (Hales et al., 2018). China is the most populous country with a total population of 1.4 billion compared to 0.32 billion in America (in 2018). Although the prevalence of obesity is much higher in America than in China, the obese population is comparable in these two countries. In 2014, China ranked first worldwide, with 43.2 million obese men and 46.4 million obese women (Collaboration, 2016).

According to the WHO classification, individuals with a body mass index (BMI) $\geq 30 \mathrm{~kg} / \mathrm{m}^{2}$ are defined as obese (WHO, 2000). However, the universal BMI criteria are not suitable among diverse Asian populations, as Asian populations show different associations between BMI, percentage of body fat, and health risks than European populations (Consultation, 2004). According to the classification of the Chinese Ministry of Public Health, Chinese individuals with a BMI $\geq 28 \mathrm{~kg} / \mathrm{m}^{2}$ are defined as obese. Waist circumference is another important indicator for obesity, which provides both independent and additive information to $\mathrm{BMI}$ for predicting morbidity and risk of death. As BMI alone is not sufficient to properly assess or manage the cardiometabolic risk, the combination of BMI and waist circumference can identify the highest-risk phenotype of obesity far better than either measure alone (Ross et al., 2020).

Obesity is the second leading cause of preventable death and has been identified as a risk factor for heart diseases, type 2 diabetes, certain types of cancers, and metabolic syndromes (Qasim et al., 2018). Overweight and obesity adversely affect cardiovascular (CV) function and serve as an independent risk factor for both systolic and diastolic heart dysfunction, resulting in heart failure (HF) (Wang and Ren, 2018). Obese women are at higher risk of CV disease (CVD), as increased aldosterone and mineralocorticoid receptor activation, aberrant estrogenic signaling, and elevated levels of androgens are among some of the proposed mechanisms explaining the heightened CVD risk. Except for the traditional CV risk factors, excess weight gain during pregnancy, preeclampsia, gestational diabetes, and menopause are central to designing personalized interventions aimed to curb the epidemic of CVD (Manrique-Acevedo et al., 2020). Maternal obesity (MO) during pregnancy exhibits intergenerational effects by programming offspring to CVD. The animal experiment indicates that MO impairs fetal cardiomyocyte contractility through altered intracellular $\mathrm{Ca}^{2+}$ handling, overloading fetal cardiomyocyte intracellular $\mathrm{Ca}^{2+}$, and aberrant myofilament protein composition (Wang Q. et al., 2019).

In obesity-related cardiomyopathy (ORCM), obesity affects the cardiac function and remodeling in the aspects of myocardial fibrosis, hemodynamic load, and impaired ventricular contractility, which eventually results in HF (Alpert et al., 2014; Carbone et al., 2017). Leptin exerts profound functions in the regulation of food intake, energy expenditure, glucose metabolism, reproduction, and immune response.
Therapeutic applications of leptin in the management of obesity and metabolic syndrome are also discussed (Zhang and Ren, 2015). Autophagy pathway may play a pivotal role in the development of cardiac anomalies induced by obesity (Xu and Ren, 2013; Zhang and Ren, 2016).

In vivo study revealed high-fat intake downregulated leptin receptor and $\operatorname{PPAR} \gamma$, insulin signaling, phosphorylation of AMPK, ACC, upregulated GATA-4, ANP, NFATc3, PPAR $\alpha$, $\mathrm{m}$-TOR/p70s6k signaling in obesity cardiomyopathy, while knocking out ET-1 receptor $\mathrm{A}\left(\mathrm{ET}_{\mathrm{A}}\right)$ attenuated the exception of AMPK/ACC. In vitro study indicated high-fat diet-induced hypertrophic and autophagic responses can be abolished by the ETA receptor antagonist (Ceylan et al., 2018). However, direct and indirect pathophysiologic factors related to obesity interplay in ORCM, the underling mechanism of ORCM had not been full studied. Meanwhile, current pharmacological treatments have limited curative effects on the clinical outcomes of ORCM. It is essential to broaden our understanding for the precise mechanisms behind these therapeutic modalities in the management of cardiometabolic diseases (Ren and Zhang, 2018). In this regard, it is urgent to explore the molecular mechanisms underlying ORCM progression and to develop novel therapeutic strategies to prevent the progression of ORCM.

Recently, the system biology analysis of microarrays has become an efficient tool for deciphering possible meaningful genes and pathophysiological pathways of various diseases. Sonne et al. (2017) performed microarray analysis to examine the gene expression profiles in adipocytes from diet-induced obese mice and obese ob/ob mice, and identified nine genes in epididymal adipocytes that are possibly involved in immune type1/type2 balance. Rendo-Urteaga et al. (2015) analyzed 28,869 genes using microarray analysis in peripheral blood mononuclear cells (PBMCs) of obese boys and suggested that changes in the gene expression profile of PBMCs in obese boys may help to understand the weight-loss response. Keustermans et al. (2017) used an Illumina microarray platform to analyze gene expression profiles in sorted monocytes from obese and lean children and revealed that monocyte gene expression in childhood obesity might be correlated with obesity and complexity of atherosclerosis in adults. In terms of cardiomyopathy, Zhao et al. (2018) analyzed the gene expression profiles (GSE3585 and GSE42955) of patients with cardiomyopathy and healthy controls and revealed that 89 differentially expressed genes (DEGs) might be associated with cardiomyopathy. In addition, Li et al. (2018) used four data profiles (GSE5406, GSE26887, GSE42955, and GSE57338) for microarray analysis and revealed a common differential gene expression signature in ischemic cardiomyopathy. However, the systematic and thorough microarray analysis for ORCM is still lacking.

In this study, we constructed a co-expression correlation network using the expression data from GSE18897 (whole blood expression profiling of obese diet-sensitive, obese dietresistant, and lean human subjects) and identified the DEGs from murine datasets (GSE47022, regular weight C57BL/6, and diet-induced obese C57BL/6 mice) using linear models for microarray (LIMMA) data. Selected co-expression modules and DEGs were further subjected to enrichment analysis of Gene 
Ontology (GO) and Kyoto Encyclopedia of Genes and Genome (KEGG) pathway. Furthermore, the hub genes derived from bioinformatics analysis were validated in the clinical samples from ORCM patients and lean controls. The present study provides preliminary insights into the molecular mechanisms underlying the progression of ORCM.

\section{MATERIALS AND METHODS}

\section{Data Collection and Processing}

The dataset (GSE18897) including 80-whole-genome expression profiles of the whole blood of obese diet-sensitive, obese dietresistant, and lean human subjects; and the dataset (GSE47022) including lean individuals and murine data, including the cardiac samples of parallel regular weight $\mathrm{C} 57 \mathrm{BL} / 6$, and diet-induced obese C57BL/6 mice, were downloaded from the GEO database. The GSE18897 series were performed on the GPL570 [HG U133_Plus_2] Affymetrix Human Genome U133 Plus 2.0 Array platform (Affymetrix, Santa Clara, CA, United States); the GSE47022 series were performed on GPL8321 [Mouse430A_2] Affymetrix Mouse Genome 430A 2.0 Array (Affymetrix). The raw data were extracted from the CEL files using the oligo package in the Bioconductor ${ }^{1}$ and subsequently processed using the robust multiarray average algorithm (Carvalho and Irizarry, 2010). The human and mouse genes were matched by Gene database ${ }^{2}$. The gene symbols of probes were annotated using the annotation profiles provided by Affymetrix. The DEGs were detected using the R package LIMMA (Ritchie et al., 2015). The cut-off criteria were set as $\log 2$-fold change $(\log 2 \mathrm{FC}) \geq 0.5$ and $P<0.05$.

\section{Construction of Co-expression Network}

Global gene expression profiles and co-expressed genes were identified using weighted gene co-expression network analysis (WGCNA; v1.49) package downloaded from Bioconductor. The possibility of two transcripts to construct a weighted network was determined using the soft-threshold method of the Pearson correlation analysis. Average linkage hierarchical clustering was performed to group transcripts based on the topological overlap dissimilarities in the network connection strengths. The restricted minimum gene number was set to 30 for each module, and a threshold of 0.25 was used to merge the similar modules.

\section{Enrichment Analysis Using GO and KEGG Pathway Analysis}

Functional enrichment analysis of the selected genes was performed using the cluster Profiler package (Ritchie et al., 2015). $P<0.05$ was considered statistically significant, and the identified significant analyses were sorted by gene counts.

\section{Protein-Protein Interaction (PPI) Network Construction}

The protein-protein interaction (PPI) network of the selected genes was constructed using the search tool for the retrieval

${ }^{1}$ http://bioconductor.org/biocLite.R

${ }^{2}$ https://www.ncbi.nlm.nih.gov/gene/ of interacting genes (STRING) database, and the threshold of medium confidence $\geq 0.4$ was applied in the analysis. The constructed PPI network was visualized using Cytoscape software $\left(\mathrm{v} 3.6 .1^{3}\right)$.

\section{Blood Sample Collection}

A total of six ORCM patients were recruited between July 2018 and June 2019 in the Sun Yat-sen Memorial Hospital of Sun Yat-sen University. The diagnosis of ORCM was based on previous reports (Lavie et al., 2013). The inclusion criteria for ORCM patients were: (1) age > 18 years old; (2) patients with $\mathrm{BMI} \geq 28 \mathrm{~kg} / \mathrm{m}^{2}$ (Chinese classification of obesity) and class II cardiac function according to the NYHA functional classification; and (3) color Doppler ultrasound examination indicating myocardial injury, including diastolic dysfunction, myocardial hypertrophy, or systolic dysfunction (EF $<60 \%$ ). Healthy volunteers (six) were recruited during routine physical examination. The inclusion criteria for healthy subjects were: (1) age > 18 years; (2) $18.5<$ BMI < 23.9; (3) no hyperlipidemia; and (4) color Doppler ultrasound examination indicating no myocardial injury. Exclusion criteria were: (1) pregnancy or breastfeeding; (2) patients with tumors or infection; and (3) patients having heart valve disease. The peripheral blood was collected from both ORCM patients and healthy controls. The serum was extracted from the peripheral blood and was stored at $-80^{\circ} \mathrm{C}$ until further analysis. All the procedures were approved by the Ethics Committee of Sun Yat-sen Memorial Hospital of Sun Yat-sen University (Ethics approval\#:SYSEC-KY-KS-2019-019), and each patient submitted written informed consent in accordance with the Declaration of Helsinki. Clinical characteristics of all patients enrolled are summarized in Tables 1, 2.

\section{Quantitative Real-Time PCR Analysis (qRT-PCR)}

The RNA from the serum was extracted using TRIzol reagent (Invitrogen, Carlsbad, CA, United States) according to the manufacturer's instructions. The mRNA was reverse transcribed into cDNA using the First Strand Synthesis kit (Thermo Fisher Scientific, Waltham, MA, United States). Real-time PCR was performed on an ABI7900 Real-time PCR system (Applied Biosystems, Foster City, CA, United States) using the SYBR Green Master Mix kit (Takara, Dalian, China). GAPDH was used as the control for mRNA expression. The relevant gene expression levels were calculated using the comparative Ct method. The primers for the relevant genes are summarized in Table 3.

\section{Enzyme-Linked Immunosorbent Assay (ELISA) Analysis}

The protein levels of elongation of very-long-chain fatty acids protein 4 (ELOVL4), matrix metalloproteinase-8 (MMP8), and interleukin-33 (IL-33) were determined by respective enzymelinked immunosorbent assay (ELISA) kits (Abcam, Cambridge, MA, United States) according to the manufacturer's instructions.

${ }^{3} \mathrm{http}: / /$ www.cytoscape.org/ 
TABLE 1 | Clinical characteristics of patients enrolled.

\begin{tabular}{lcc}
\hline Characteristics & ORCM $(\boldsymbol{N}=\mathbf{6})$ & Control $(\boldsymbol{n}=\mathbf{6})$ \\
\hline Age (years) & $51.8 \pm 14.8$ & $51.5 \pm 10.5$ \\
Men $(N, \%)$ & $5(83.3 \%)$ & $3(50 \%)$ \\
Women $(N, \%)$ & $1(16.7 \%)$ & $3(50 \%)$ \\
CAD $(N, \%)$ & $1(16.6 \%)$ & $0(0 \%)$ \\
Hypertension (N,\%) & $2(33.3 \%)$ & $0(0 \%)$ \\
Diabetes (N,\%) & $0(0 \%)$ & $0(0 \%)$ \\
BMI (kg/m $\left.{ }^{2}\right)$ & $30.9 \pm 3.5$ & $24.2 \pm 3.5$ \\
Echocardiography parameters & & \\
LA (mm) & $40 \pm 4.4$ & $35 \pm 4.9$ \\
LVDd (mm) & $58.1 \pm 5.4$ & $49.3 \pm 5.0$ \\
IVSTd (mm) & $10.5 \pm 1.5$ & $8.8 \pm 1.1$ \\
EF (\%) & $54.8 \pm 16.7$ & $70.3 \pm 2.8$ \\
Diastolic dysfunction $(N, \%)$ & $2(33.3 \%)$ & $0(0 \%)$ \\
NYHA grading & & \\
Grade I $(N, \%)$ & $2(33.3 \%)$ & $6(100 \%)$ \\
Grade II (N,\%) & $4(66.7 \%)$ & $0(0 \%)$ \\
Grade III (N,\%) & $(0 \%)$ & $0(0 \%)$ \\
Grade IV (N,\%) & $(0 \%)$ & $0(0 \%)$
\end{tabular}

Data are present as mean $\pm S D$; $C A D$, coronary artery disease; $B M I$, body mass index; $E F$, ejection fraction; $L A$, left atrial; $L V D d$, left ventricular end diastolic diameter; IVSTd, intel-ventricular scptum end-diastolic thickness; EF, ejection fraction.

\section{Statistical Analysis}

qRT-PCR and ELISA data were analyzed using GraphPad Prism 5 (GraphPad Software, La Jolla, CA, United States). The data were presented as mean \pm standard deviation. Significant differences between groups were analyzed using unpaired $t$-test. ${ }^{*} P<0.05$ was considered statistically significant.

\section{RESULTS}

\section{The Workflow of Study Strategies}

Figure 1 illustrates the workflow of the experimental design and data processing. One microarray-based gene expression dataset
TABLE 3 | Primer sequences for qRT-PCR.

\begin{tabular}{|c|c|c|}
\hline Genes & Forward $\left(5^{\prime}-3^{\prime}\right)$ & Reverse $\left(5^{\prime}-3^{\prime}\right)$ \\
\hline & GCTCGGGCTACATCACCATC & GGTCCCGGCTTTCGTTCAG \\
\hline CD45 & ACCACAAGTTTACTAACGCAAGT & TTTAGGGGGATTCCAGGTAA \\
\hline 33 & ATGGCTGAGGGATCAGTGATG & CCAGATTGCCGTAGTTCT \\
\hline & ААTTТACTTGT & गाTGGTGGGAGCTGATACAGG \\
\hline MMP8 & TGCTCTTACTCCATGTGCAGA & TCCAGGTAGTCCTGAACAGTTI \\
\hline CYPB & AAGGTGTATIT & TAGCCAAATCO \\
\hline ELOVL4 & GAGCCGGGTAGTGTCCTAAAC & CACACGCTTATCTGCGATGG \\
\hline SNCA & AAGAGGGTGTTCTCTATGTAGGC & GСTCCTCCAACATTTGTCACTT \\
\hline IL33 & GTGACGGTGTTGATGGTAAGAT & AGCTCCACAGAGTGTTCCTTG \\
\hline GAPDH & AGGTGAAGGTCGGAGTCAAC & CGCTCCTGGAAGATGGTGAT \\
\hline
\end{tabular}

(GSE18897) included 80-whole-genome expression profiling of the whole blood from obese diet-sensitive, obese diet-resistant, and lean human subjects. The human datasets were used for constructing gene networks with WGCNA, and the expression models were subjected to GO term enrichment and KEGG pathway enrichment analysis. The top highest degree genes were chosen to overlap with the murine heart DEGs. The murine GSE47022 datasets include the cardiac samples of parallel regular weight C57BL/6 and diet induced obese C57BL/6 mice. The GSE47022 datasets were processed using LIMMA to reveal the DEGs in the heart samples. In addition, the overlap hub genes were verified in the clinical samples.

\section{Construction and Analysis of Gene Co-expression Network With DEGs in Obese Individuals}

The co-expression network of 5121 genes was analyzed using WGCNA to explore the gene expression network in obesity. First, as shown in Figure 2A, sample clustering was undertaken to detect outliers. The sample clusters were summarized based on the flash-cluster method by combining a heuristic cut-off, and the top $25 \%$ most variably expressed genes were selected for analysis (Figure $\mathbf{2 A}$ ). The power value is a critical parameter and

TABLE 2 | Anthropometric information of patient enrolled.

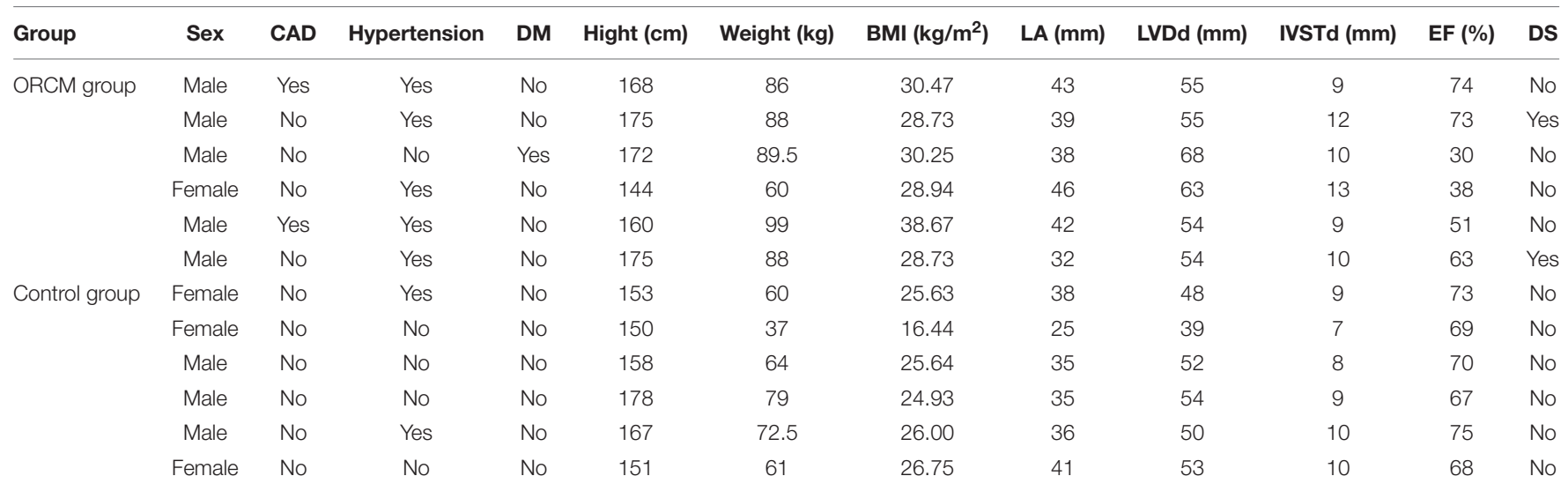

CAD, coronary artery disease; DM, diabetes mellitus; BMI, body mass index; LA, left atrial; LVDd, left ventricular end diastolic diameter; IVSTd, intel-ventricular scptum end-diastolic thickness; EF, ejection fraction; DS, diastolic dysfunction. 


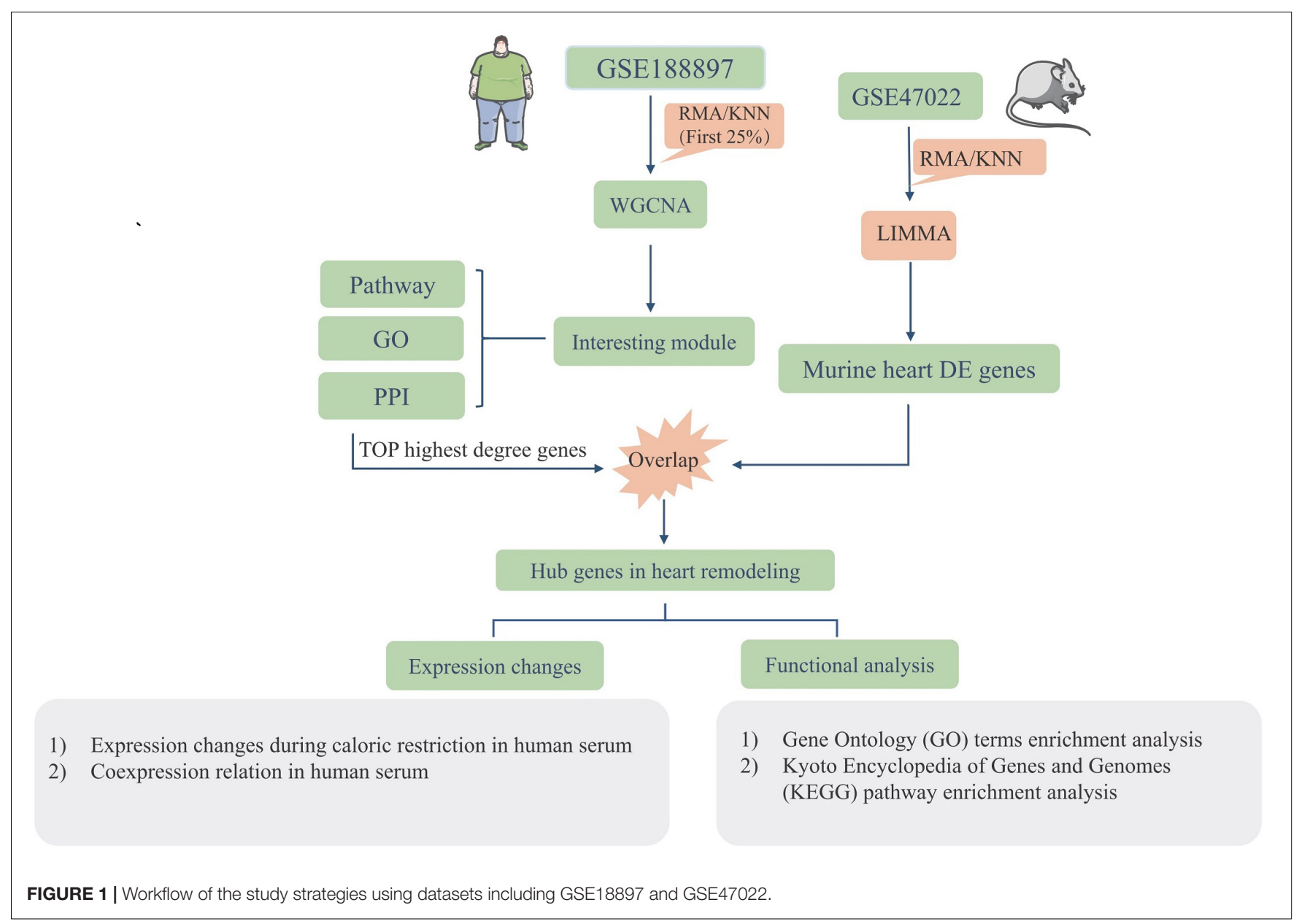

affects the average connectivity degree and independence of the co-expression modules. In this regard, we screened the network topology using different soft thresholding powers, and $\beta=16$ was chosen for further analysis (Figure 2B). Provided that the appropriate soft power parameter was chosen, the eigengenes were used as representative profiles to quantify module similarity by the eigengene correlation (Figure 2C).

Figure $3 \mathrm{~A}$ demonstrates the construction of co-expression modules with no merge and merge cut height at 0.25 , and the WGCNA analysis, which produced a hierarchical clustering tree (dendrogram) of 5121 genes. A total of eight co-expression modules were generated based on the analysis (Figure 3B). The smallest co-expression module contained 50 genes, while the largest co-expression module contained 3000 genes, and on average, each co-expression module contained 1250 genes. We further determined if any co-expression module was associated with the caloric restriction treatment and investigated the relevance between each module and traits of the caloric restriction treatment. Among eight modules, the co-expression module significance of the yellow co-expression module was higher than that of any other, suggesting it had a greater correlation with the caloric restriction treatment (Figure 3B). The yellow co-expression module showed a positive correlation with the caloric restriction treatment $(r=0.74, p=4 \mathrm{e}-7)$ and was chosen for further investigation. GO function and KEGG pathway enrichment analyses were performed using DAVID functional annotation to determine the function of these genes in the yellow module. For GO biological processes, genes in the co-expression module were significantly enriched in oxygen transport (Figure 3D); for the KEGG analysis, the genes were mainly enriched in the metabolism of porphyrins (Figure 3D) and the datas can be found in Supplementary Tables S1, S2 in Supplementary Material.

The yellow co-expression module genes were used on the STRING database to clarify high confidence hub genes. The genes were ranked by the PPI nodes, and the top 50 genes were selected (Figure 3E). Among these genes, FECH, SNCA, GLRX5, BPGM, SENSN, BNIP3L, and RAB2B showed the highest connectivity (Figures 3C,E).

\section{Overlap Analysis of Hub Genes From Human and Mouse DEGs}

The genes in the yellow co-expression module and DEGs from the murine datasets were subjected to KEGG pathway enrichment analysis and the datas can be found in Supplementary Table S3 in Supplementary Material. As shown in Figure 4A, for the murine datasets, metabolic, AMPK, 


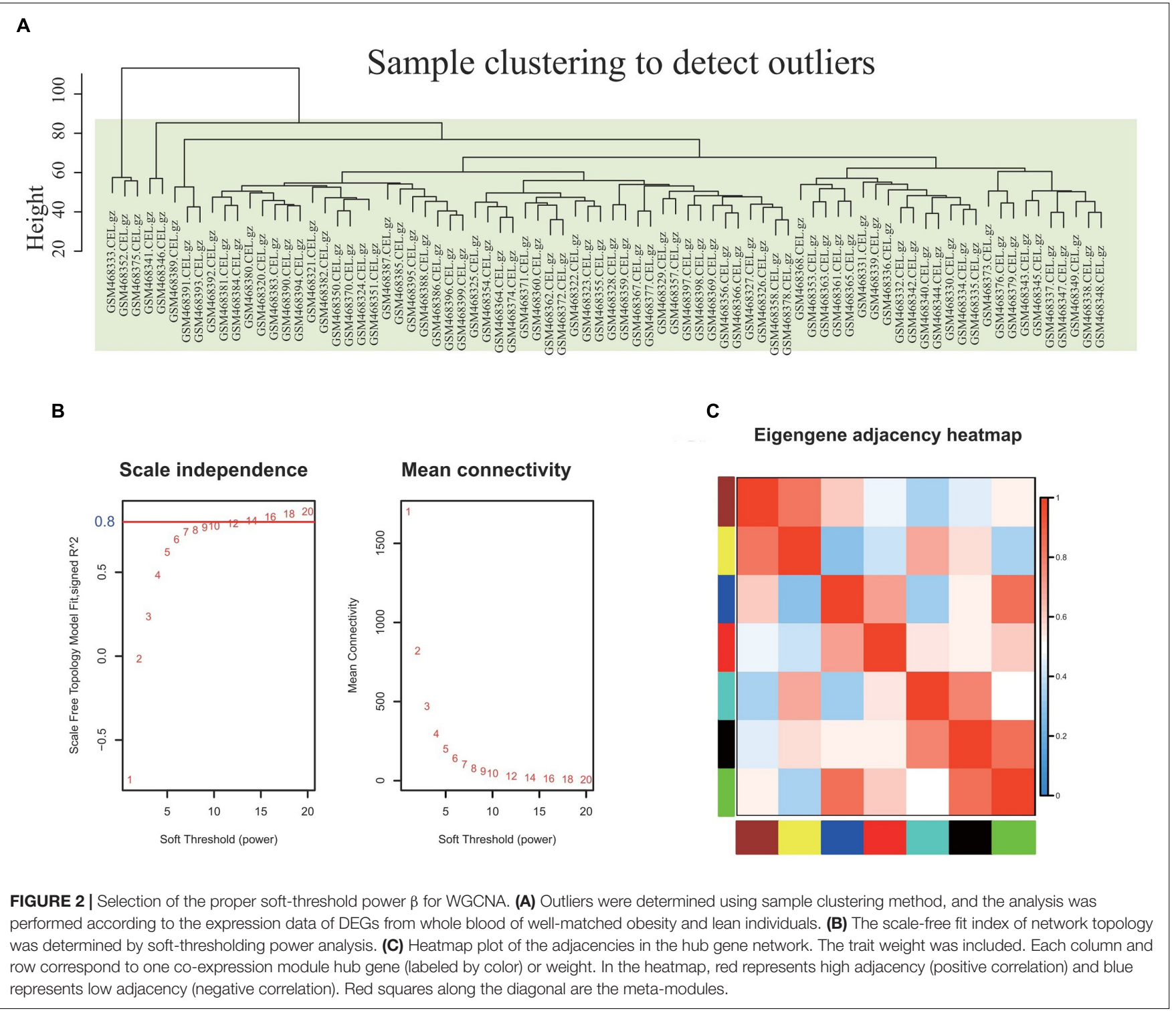

fatty acid, PPAR signaling, insulin signaling, biosynthesis of antibiotics, biosynthesis of unsaturated fatty acids, glucagon signaling, insulin resistance pathways were enriched; for the yellow co-expression module genes, Alzheimer's disease, MAPK signaling, oxidative phosphorylation, ABC transporters cardiac muscle contraction signaling pathways were enriched. The overlap showed that six common hub genes (ELOVL4, CYPB, SNCA, ZFN383, MMP8, and IL-33) were detected (Figure 4B). The volcano plot results showed that the six common hub genes were all upregulated (Figure 4C).

\section{Validation of Hub Genes in the Human Clinical Samples}

To validate the results from bioinformatics analysis, we examined the gene expression levels of these hub genes in human peripheral blood from patients with ORCM and healthy controls. As shown in Figure 5A, the expression levels of ELOVL4, MMP8, and IL-33 were significantly upregulated in the ORCM group compared to normal controls, while no significant difference was detected in the other hub genes. Furthermore, the increased protein levels of ELOVL4, MMP8, and IL-33 in the peripheral blood from patients with ORCM were confirmed using ELISA (Figure 5B).

\section{Correlation Analysis of ELOVL4 and IL33 Gene Expression Levels Using GEO Database}

The KEGG pathway analysis revealed that the hub genes derived from the overlap analysis were involved in the fatty acid metabolism and IL33/sST2 signaling pathways (Figure 6A). The correlation analysis showed that ELOVL4 expression levels were positively correlated with IL-33 expression levels (Figure 6B). More importantly, analysis of ELOLV4 and IL-33 using GEO 
A

B

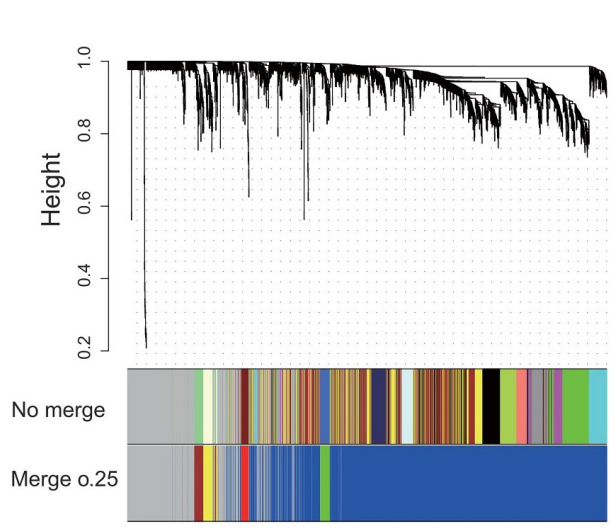

D

D

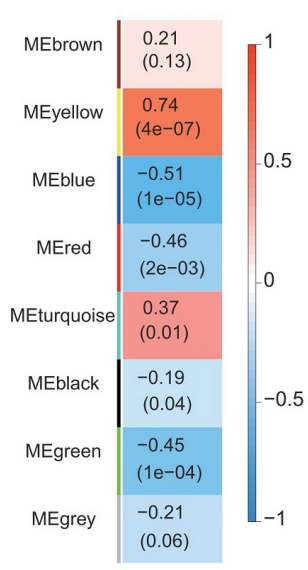

C

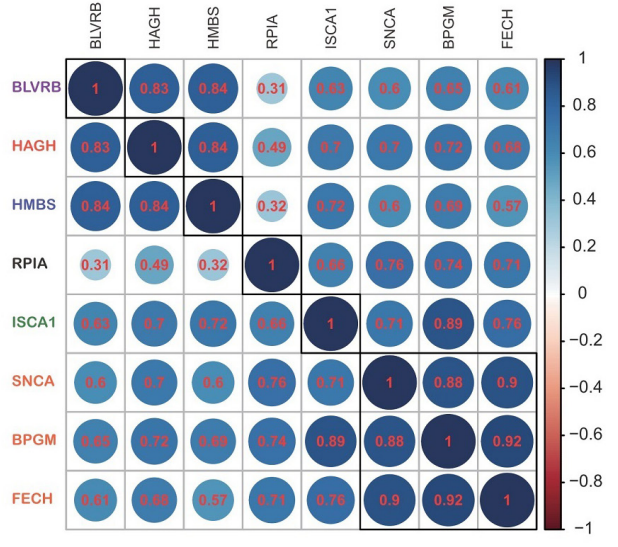

E
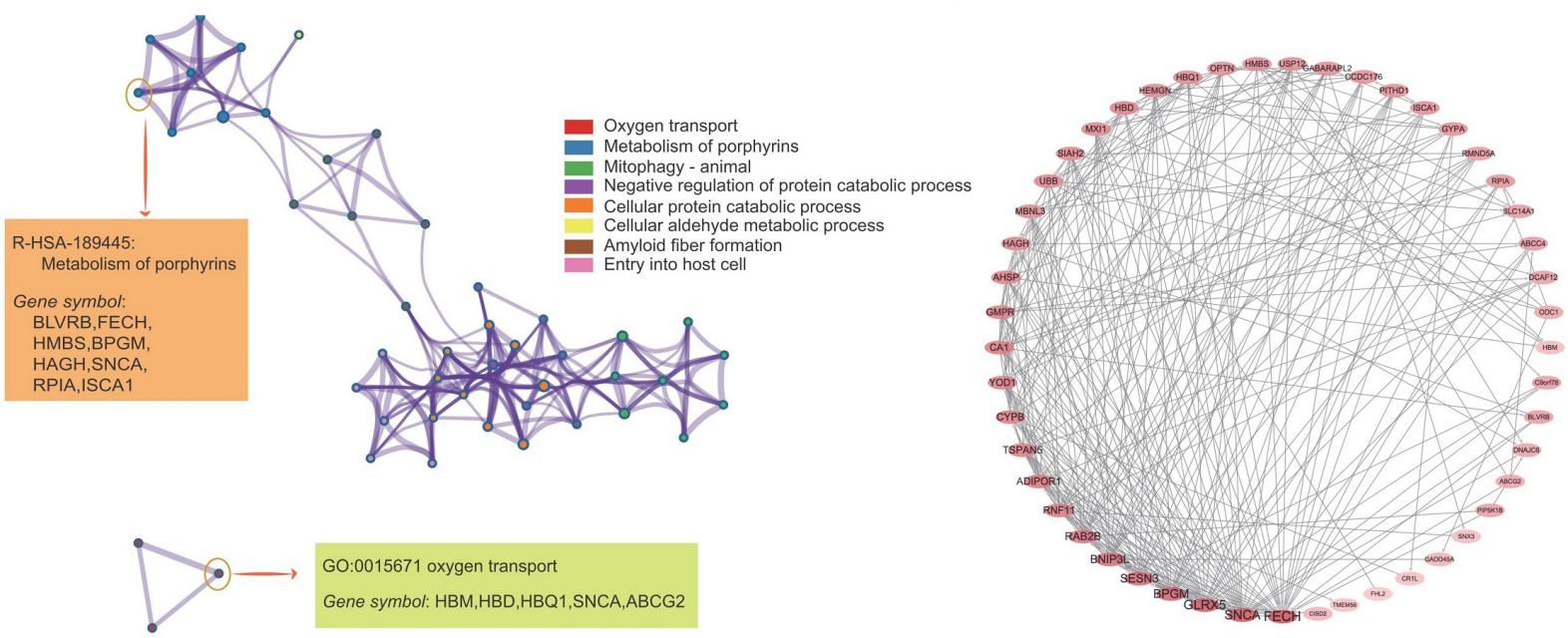

FIGURE 3 | Construction of the co-expression network and identification of the most related co-expression modules. (A) Clustering of genes together with assigned module colors. The dissimilarity was based on the topological overlap. The $y$-axis is the distance determined by the extent of the topological overlap. (B) Heatmap with each cell containing the $p$-value correlation from linear mixed effects model. Row corresponds to different co-expression modules; column corresponds to traits of caloric restriction treatment. (C) The gene correlation heatmap of the genes in yellow co-expression module. Each color circle represents the distance between different genes. (D) GO enrichment and KEGG pathway analysis of 633 genes in the yellow co-expression module. (E) PPI network construction of the top 50 genes in the yellow co-expression module.

database showed that ELOVL4 and IL33 expression were both upregulated in the obese group when compared to the lean group. Caloric restriction for 3 and 6 months significantly attenuated the increased expression levels of ELOVL4 in the obesity group, while a significant reduction of IL-33 expression levels was observed in the obese patients with a 6 -month period of caloric restriction treatment (Figure 6C).

\section{DISCUSSION}

The molecular mechanisms underlying ORCM progression involve multiple signaling pathways and the pharmacological treatment for ORCM is still limited (Khan and Movahed, 2013; Alpert et al., 2014; Tiwari and Ndisang, 2014; Bhatheja et al., 2016). Thus, it is necessary to explore new targets and develop novel therapies. Microarray analysis for gene expression profiles using different bioinformatics tools has been an effective strategy for identifying novel targets for various diseases. In the present study, we performed WGCNA on the series GSE188897 combined with routine bioinformatics analysis and revealed six potential target genes that might be involved in the development of obesity. In addition, the DEGs from GSE47022 were analyzed. The overlap analysis of the hub genes from the WGCNA analysis of GSE188897 and DEGs from GSE47022 revealed six potential genes that may be associated with ORCM. qRT-PCR and ELISA 
A

B

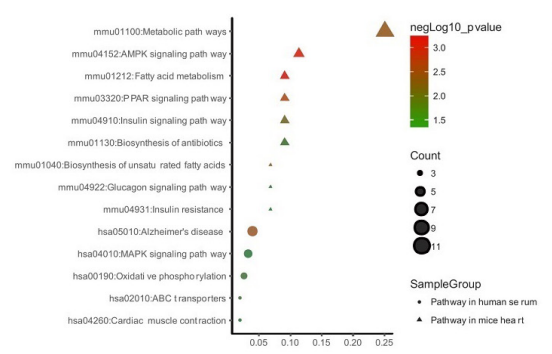

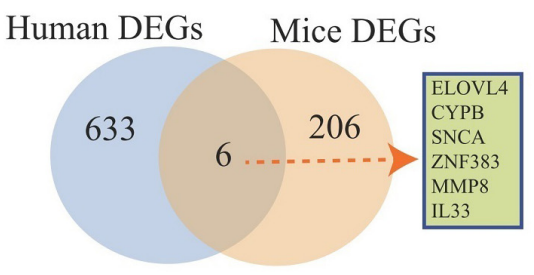

C

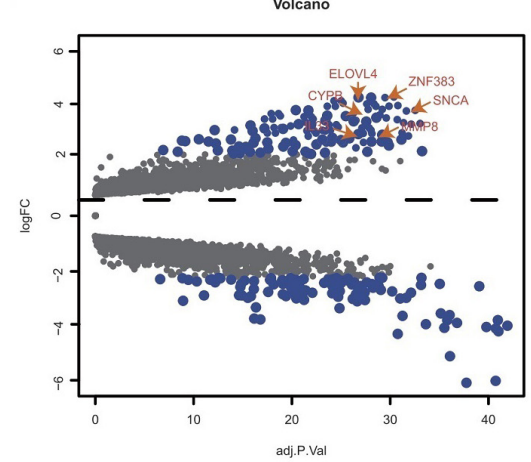

FIGURE 4 | Overlap analysis of hub genes from human and mouse DEGs. (A) KEGG pathway analysis of genes from yellow co-expression module and DEGs from mice. (B) Venn diagram shows common hub genes between yellow co-expression module and DEGs from mice. (C) Volcano plot of yellow co-expression module and DEGs from murine datasets.
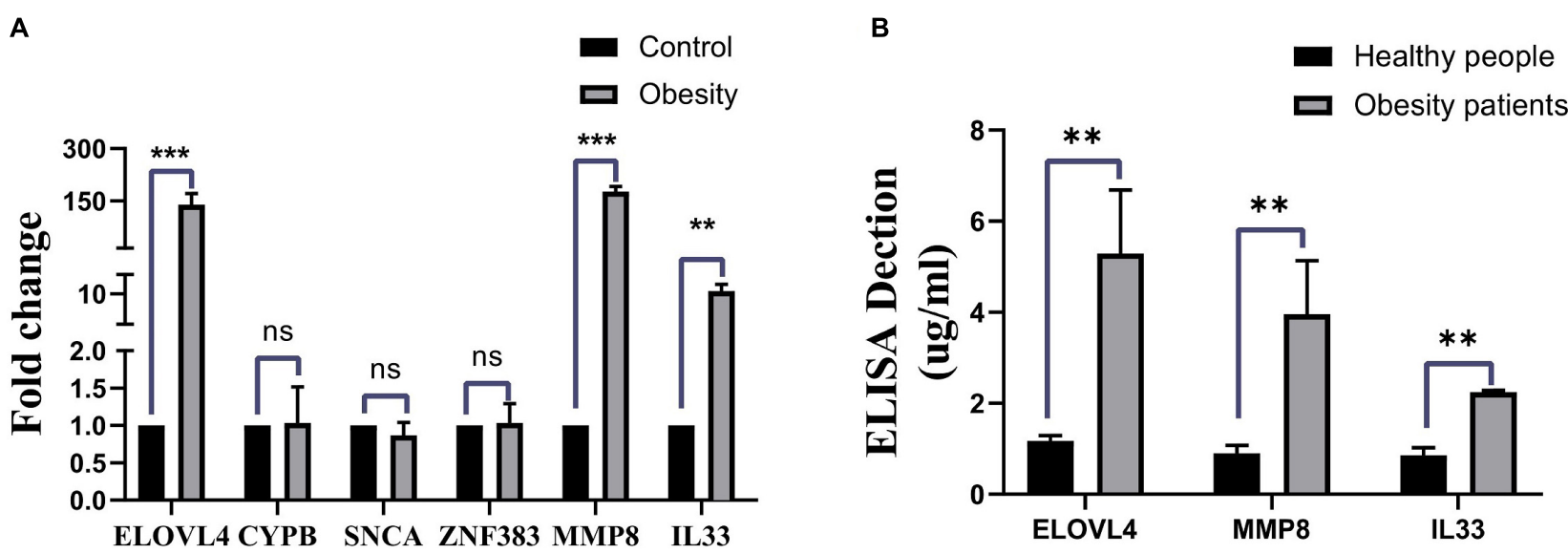

FIGURE 5 | Validation of hub genes in the clinical human samples. (A) Gene expression levels of the hub genes (ELOVL4, CYPB, SNCA, ZFN383, MMP8, and IL33) in the human peripheral blood from ORCM patients and healthy controls were determined by qRT-PCR. (B) Protein levels of ELOVL4, MMP8, and IL33 in the human peripheral blood from ORCM patients and healthy controls were determined by ELISA assay. $N=6$; ns = non-significant; ${ }^{* *} P<0.01$.

further validated the upregulation of ELOVL4, MMP8, and IL33 in the patients with ORCM when compared to healthy lean controls. Collectively, in the present study, we used different bioinformatics analytical strategies to explore the potential hub genes related to ORCM development. Further validation studies indicated that ELOVL4, MMP8, and IL-33 might participate in the progression of ORCM.

WGCNA is a newly developed method to identify highly correlated genes in microarrays (Langfelder and Horvath, 2008; Pei et al., 2017). In contrast with the traditional gene-gene correlation co-expression matrices by setting a hard threshold, WGCNA can identify correlated genes using a soft-threshold algorithm and determine the relationship between co-expression modules and phenotypes (Kakati et al., 2019). Thus, WGCNA has been widely used in the study of various diseases, including CVDs (Chen et al., 2016; Wang Y. et al., 2019). Kang et al. (2020) performed the WGCNA in GSE79962 and revealed the potential critical roles of NDUFB5, TIMMDC1, and VDAC3 in the septic cardiomyopathy progression. Using microarray data of coronary artery diseases (CADs; GSE23561), studies detected a co-expression module associated with hypertrophic cardiomyopathy pathway in CAD and found that G6PD and S100A7 were the potential targets (Liu et al., 2016). The GSE18897 datasets were originally generated by Ghosh et al. (2010), and further pathway analysis by gene-set enrichment showed increased transcript levels for genes classified in the oxidative phosphorylation, apoptosis, and ribosome pathways in the obese cohort. In the present study, WGCNA was performed in GSE18897 datasets, and yellow co-expression module was chosen for further GO function and KEGG pathway enrichment analyses. GO function enrichment revealed the genes related to oxygen transport, which was consistent with previous studies showing that transcripts associated with oxygen transport were elevated with the increasing BMI (Rai et al., 2014). Moreover, the genes related to oxygen transport are also upregulated in the peripheral blood from patients with pulmonary hypertension (Cheadle et al., 2012), suggesting the potential involvement of these genes in the ORCM. The KEGG pathway analysis 
A

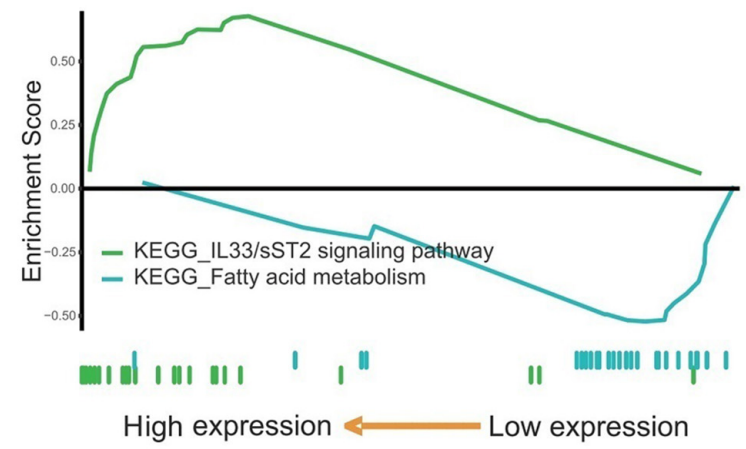

B

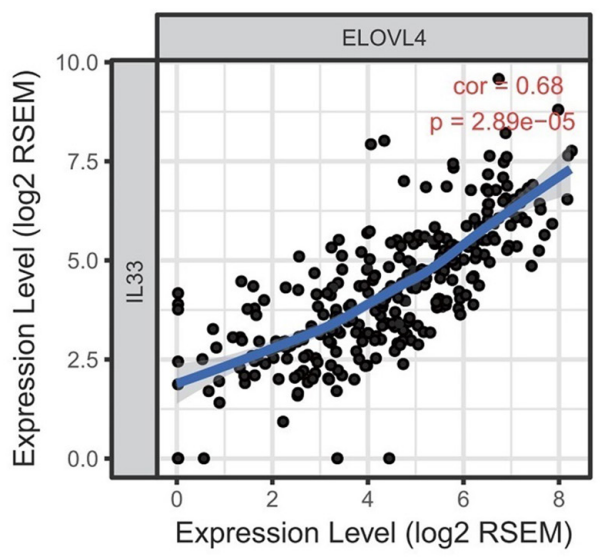

C
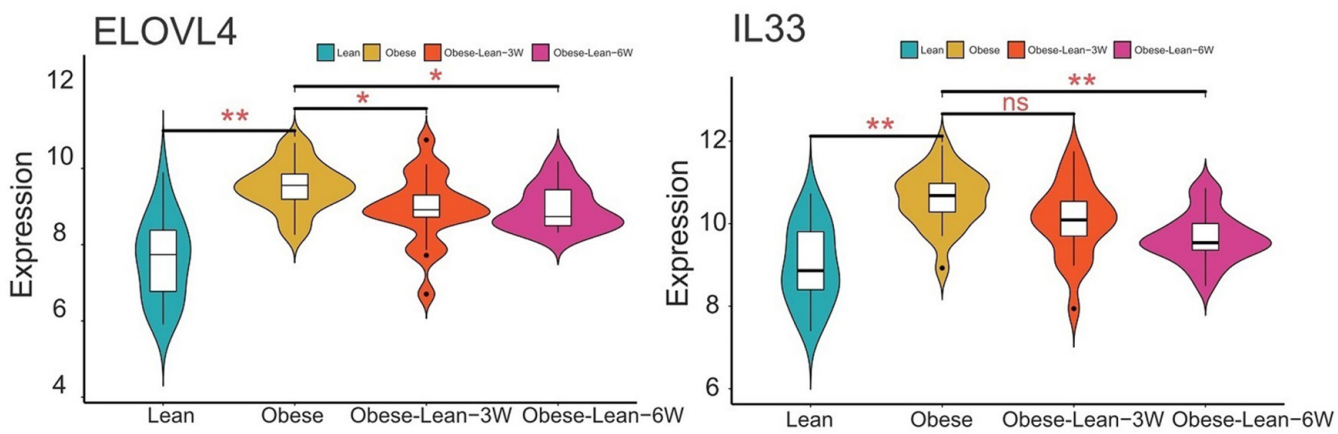

FIGURE 6 | Correlation analysis of ELOVL4 and IL33 gene expression levels. (A) KEGG pathway analysis of the hub genes. (B) The correlation between ELOVL4 and IL33 expression levels were analyzed using Pearson correlation analysis. (C) Analysis of ELOVL4 and IL33 expression levels in lean controls, obese patients, and obese patients received 3- or 6-month period of caloric restriction treatment.

identified enriched genes related to porphyrin metabolism. The porphyrins metabolism is associated with the heme biosynthesis, which is functionally linked to adipogenesis via mitochondrial respiratory activity (Moreno-Navarrete et al., 2017). Our analysis strategies enabled us to identify important genes related to obesity development.

Overlap analysis of hub genes from the yellow co-expression module and DEGs from the murine datasets revealed six upregulated genes. Further experimental validation results showed that ELOVL4, MMP8, and IL-33 were upregulated in the peripheral blood from patients with ORCM compared to the lean controls. The bioinformatics analysis revealed that ELOVL4 expression levels were positively correlated with IL-33 expression levels. These data suggest the potential involvement of ELOVL4 and IL-33 in the pathophysiology of ORCM. ELOVL4 is an elongase that participates in the biosynthesis of very-long-chain (VLC, $\geq \mathrm{C} 28$ ) saturated fatty acid (VLC-SFA) and polyunsaturated fatty acid (VLC-PUFA) in different tissues (Hopiavuori et al., 2019), and plays a key role in the retinal function (Harkewicz et al., 2012). The increased deposition of long-chain fatty acids in adipocytes is one of the main characteristics of obesity (Walewski et al.,
2010). In addition, ELOVL4 is involved in the biosynthesis of VLC fatty acids in the cardiomyocytes (Agbaga et al., 2008). Ge et al. (2012) demonstrated that accumulation of the cardiomyocyte triglyceride and reduction in the ventricular function in obese mice reflected enhanced VLC fatty acid uptake and de novo fatty acid synthesis. The involvement of ELOV4 in ORCM might be due to its regulatory effects on the VLC fatty acid synthesis. IL-33 belongs to the IL-1 family of cytokines and acts as an ST2 receptor ligand, and the IL-33/ST2 signaling plays an important role in regulating the progression of cardiomyopathy (Demyanets et al., 2013). Upregulation of IL-33 has been detected in the obese adipose tissues (de Oliveira et al., 2019). Nevertheless, the interaction between ELOVL4 and IL-33 has not been determined, which requires further investigation.

\section{LIMITATIONS}

There are several limitations to this study. First, the clinical sample size for validation is relatively small, and future studies are required to increase the sample size and recruit human subjects from multiple clinical centers to confirm the findings. Second, 
the microarray data were retrieved from diet-induced obese mice, and whether the obese mice had cardiomyopathy is not known, which may lessen the significance of our findings. In terms of the database selection, only one database was chosen for the data analysis, which may have the selection bias, and future studies should consider including more datasets to validate our current findings. Third, whether the caloric restriction treatment in patients with ORCM could restore the expression levels of ELOVL4 and IL-33 requires further determination. Finally, the present study lacks the mechanistic studies regarding the role of ELOVL4 and IL-33 in the ORCM pathophysiology, which may be considered in the future investigation.

\section{CONCLUSION}

Using WGCNA in combination with integrated bioinformatics analysis, the hub genes of ELVOL4 and IL-33 might serve as potential biomarkers for diagnosis and/or therapeutic targets for ORCM. The detailed roles of ELVOL4 and IL-33 in the pathophysiology of ORCM still require further investigation.

\section{DATA AVAILABILITY STATEMENT}

The datasets generated for this study can be found in the Gene Expression Omnibus (accession: GSE18897, GSE47022).

\section{ETHICS STATEMENT}

The studies involving human participants were reviewed and approved by the Ethics Committee of Sun Yat-sen Memorial

\section{REFERENCES}

Agbaga, M. P., Brush, R. S., Mandal, M. N., Henry, K., Elliott, M. H., and Anderson, R. E. (2008). Role of Stargardt-3 macular dystrophy protein (ELOVL4) in the biosynthesis of very long chain fatty acids. Proc. Natl. Acad. Sci. U.S.A. 105, 12843-12848. doi: 10.1073/pnas.0802607105

Alpert, M. A., Lavie, C. J., Agrawal, H., Aggarwal, K. B., and Kumar, S. A. (2014). Obesity and heart failure: epidemiology, pathophysiology, clinical manifestations, and management. Transl. Res. 164, 345-356. doi: 10.1016/j.trsl. 2014.04.010

Bhatheja, S., Panchal, H. B., Ventura, H., and Paul, T. K. (2016). Obesity cardiomyopathy: pathophysiologic factors and nosologic reevaluation. Am. J. Med. Sci. 352, 219-222. doi: 10.1016/j.amjms.2016.05.014

Carbone, S., Lavie, C. J., and Arena, R. (2017). Obesity and heart failure: focus on the obesity paradox. Mayo. Clin. Proc. 92, 266-279. doi: 10.1016/j.mayocp.2016. 11.001

Carvalho, B. S., and Irizarry, R. A. (2010). A framework for oligonucleotide microarray preprocessing. Bioinformatics 26, 2363-2367. doi: 10.1093/ bioinformatics/btq431

Ceylan, A. F., Wang, S., Kandadi, M. R., Chen, J., Hua, Y., Pei, Z., et al. (2018). Cardiomyocyte-specific knockout of endothelin receptor a attenuates obesity cardiomyopathy. Biochim. Biophys. Acta Mol. Basis Dis. 1864, 3339-3352. doi: 10.1016/j.bbadis.2018.07.020

Cheadle, C., Berger, A. E., Mathai, S. C., Grigoryev, D. N., Watkins, T. N., Sugawara, Y., et al. (2012). Erythroid-specific transcriptional changes in PBMCs from pulmonary hypertension patients. PLoS One 7:e34951. doi: 10.1371/journal. pone.0034951
Hospital of Sun Yat-sen university. The patients/participants provided their written informed consent to participate in this study. Written informed consent was obtained from the individual(s) for the publication of any potentially identifiable images or data included in this article.

\section{AUTHOR CONTRIBUTIONS}

JZ and SL contributed to study conception and design. JT and YW performed the experiments and analyzed the data. LL interpreted the results. JT prepared figures and drafted the manuscript. All authors edited and revised the manuscript.

\section{FUNDING}

This work was supported by grants from Youth Program of National Natural Science Foundation of China (No. 81702618).

\section{ACKNOWLEDGMENTS}

We would like to thank our colleagues, especially Chang Liu, for his help in collecting the blood samples.

\section{SUPPLEMENTARY MATERIAL}

The Supplementary Material for this article can be found online at: https://www.frontiersin.org/articles/10.3389/fphys. 2020.00542/full\#supplementary-material

Chen, J., Yu, L., Zhang, S., and Chen, X. (2016). Network analysis-based approach for exploring the potential diagnostic biomarkers of acute myocardial infarction. Front. Physiol. 7:615. doi: 10.3389/fphys.2016.00615

Collaboration, N. C. D. R. F. (2016). Trends in adult body-mass index in 200 countries from 1975 to 2014: a pooled analysis of 1698 population-based measurement studies with 19.2 million participants. Lancet 387, 1377-1396. doi: 10.1016/S0140-6736(16)30054-X

Consultation, W. H. O. E. (2004). Appropriate body-mass index for Asian populations and its implications for policy and intervention strategies. Lancet 363, 157-163. doi: 10.1016/S0140-6736(03)15268-3

de Oliveira, M. F. A., Talvani, A., and Rocha-Vieira, E. (2019). IL-33 in obesity: where do we go from here? Inflamm. Res. 68, 185-194. doi: 10.1007/s00011019-01214-2

Demyanets, S., Kaun, C., Pentz, R., Krychtiuk, K. A., Rauscher, S., Pfaffenberger, S., et al. (2013). Components of the interleukin-33/ST2 system are differentially expressed and regulated in human cardiac cells and in cells of the cardiac vasculature. J. Mol. Cell Cardiol. 60, 16-26. doi: 10.1016/j.yjmcc.2013.03.020

Ge, F., Hu, C., Hyodo, E., Arai, K., Zhou, S., Lobdell, H. T., et al. (2012). Cardiomyocyte triglyceride accumulation and reduced ventricular function in mice with obesity reflect increased long chain Fatty Acid uptake and de novo Fatty Acid synthesis. J. Obes. 2012:205648. doi: 10.1155/2012/205648

Ghosh, S., Dent, R., Harper, M. E., Gorman, S. A., Stuart, J. S., and McPherson, R. (2010). Gene expression profiling in whole blood identifies distinct biological pathways associated with obesity. BMC Med. Genomics 3:56. doi: 10.1186/17558794-3-56

Hales, C. M., Fryar, C. D., Carroll, M. D., Freedman, D. S., and Ogden, C. L. (2018). Trends in obesity and severe obesity prevalence in US youth and adults by sex 
and age, 2007-2008 to 2015-2016. JAMA 319, 1723-1725. doi: 10.1001/jama. 2018.3060

Harkewicz, R., Du, H., Tong, Z., Alkuraya, H., Bedell, M., Sun, W., et al. (2012). Essential role of ELOVL4 protein in very long chain fatty acid synthesis and retinal function. J. Biol. Chem. 287, 11469-11480. doi: 10.1074/jbc.M111. 256073

He, Y., Pan, A., Wang, Y., Yang, Y., Xu, J., Zhang, Y., et al. (2017). Prevalence of overweight and obesity in 15.8 million men aged $15-49$ years in rural China from 2010 to 2014. Sci. Rep 7:5012. doi: 10.1038/s41598-017-04135-4

Hopiavuori, B. R., Anderson, R. E., and Agbaga, M. P. (2019). ELOVL4: Very longchain fatty acids serve an eclectic role in mammalian health and function. Prog. Retin. Eye Res. 69, 137-158. doi: 10.1016/j.preteyeres.2018.10.004

Kakati, T., Bhattacharyya, D. K., Barah, P., and Kalita, J. K. (2019). Comparison of methods for differential co-expression analysis for disease biomarker prediction. Comput. Biol. Med. 113:103380. doi: 10.1016/j.compbiomed.2019. 103380

Kang, K., Li, J., Li, R., Xu, X., Liu, J., Qin, L., et al. (2020). Potentially critical Roles of NDUFB5, TIMMDC1, and VDAC3 in the progression of septic cardiomyopathy through integrated bioinformatics analysis. DNA Cell Biol. 39, 105-117. doi: 10.1089/dna.2019.4859

Keustermans, G. C., Kofink, D., Eikendal, A., de Jager, W., Meerding, J., Nuboer, R., et al. (2017). Monocyte gene expression in childhood obesity is associated with obesity and complexity of atherosclerosis in adults. Sci. Rep. 7:16826. doi: 10.1038/s41598-017-17195-3

Khan, M. F., and Movahed, M. R. (2013). Obesity cardiomyopathy and systolic function: obesity is not independently associated with dilated cardiomyopathy. Heart Fail Rev. 18, 207-217. doi: 10.1007/s10741-012-9320-4

Langfelder, P., and Horvath, S. (2008). WGCNA: an R package for weighted correlation network analysis. BMC Bioinformatics 9:559. doi: 10.1186/14712105-9-559

Lavie, C. J., Alpert, M. A., Arena, R., Mehra, M. R., Milani, R. V., and Ventura, H. O. (2013). Impact of obesity and the obesity paradox on prevalence and prognosis in heart failure. JACC Heart Fail 1, 93-102. doi: 10.1016/j.jchf.2013.01.006

Li, Y., Jiang, Q., Ding, Z., Liu, G., Yu, P., Jiang, G., et al. (2018). Identification of a common different gene expression signature in ischemic cardiomyopathy. Genes (Basel) 9:56. doi: 10.3390/genes9010056

Liu, J., Jing, L., and Tu, X. (2016). Weighted gene co-expression network analysis identifies specific modules and hub genes related to coronary artery disease. BMC Cardiovasc. Disord. 16:54. doi: 10.1186/s12872-016-0217-3

Manrique-Acevedo, C., Chinnakotla, B., Padilla, J., Martinez-Lemus, L. A., and Gozal, D. (2020). Obesity and cardiovascular disease in women. Int. J. Obes. (Lond.) 2020. doi: 10.1038/s41366-020-0548-0

Moreno-Navarrete, J. M., Rodriguez, A., Ortega, F., Becerril, S., Girones, J., SabaterMasdeu, M., et al. (2017). Heme biosynthetic pathway is functionally linked to adipogenesis via mitochondrial respiratory activity. Obesity (Silver Spring) 25, 1723-1733. doi: 10.1002/oby.21956

Pei, G., Chen, L., and Zhang, W. (2017). WGCNA application to proteomic and metabolomic data analysis. Methods Enzymol. 585, 135-158. doi: 10.1016/bs. mie.2016.09.016

Qasim, A., Turcotte, M., de Souza, R. J., Samaan, M. C., Champredon, D., Dushoff, J., et al. (2018). On the origin of obesity: identifying the biological, environmental and cultural drivers of genetic risk among human populations. Obes. Rev. 19, 121-149. doi: 10.1111/obr.12625

Rai, M. F., Patra, D., Sandell, L. J., and Brophy, R. H. (2014). Relationship of gene expression in the injured human meniscus to body mass index: a biologic connection between obesity and osteoarthritis. Arthr. Rheumatol. 66, 2152-2164. doi: $10.1002 /$ art.38643

Ren, J., and Zhang, Y. (2018). New therapeutic approaches in the management of cardiometabolic diseases: bringing the concepts together. Curr. Drug Targets 19, 987-988. doi: 10.2174/138945011909180629095709
Rendo-Urteaga, T., Garcia-Calzon, S., Gonzalez-Muniesa, P., Milagro, F. I., Chueca, M., Oyarzabal, M., et al. (2015). Peripheral blood mononuclear cell gene expression profile in obese boys who followed a moderate energy-restricted diet: differences between high and low responders at baseline and after the intervention. Br. J. Nutr. 113, 331-342. doi: 10.1017/ S0007114514003584

Ritchie, M. E., Phipson, B., Wu, D., Hu, Y., Law, C. W., Shi, W., et al. (2015). limma powers differential expression analyses for RNA-sequencing and microarray studies. Nucleic Acids Res. 43:e47. doi: 10.1093/nar/gkv007

Ross, R., Neeland, I. J., Yamashita, S., Shai, I., Seidell, J., Magni, P., et al. (2020). Waist circumference as a vital sign in clinical practice: a consensus Statement from the IAS and ICCR working group on visceral obesity. Nat. Rev. Endocrinol. 16, 177-189. doi: 10.1038/s41574-019-0310-7

Sonne, S. B., Yadav, R., Yin, G., Dalgaard, M. D., Myrmel, L. S., Gupta, R., et al. (2017). Obesity is associated with depot-specific alterations in adipocyte DNA methylation and gene expression. Adipocyte 6, 124-133. doi: 10.1080/21623945. 2017.1320002

Tiwari, S., and Ndisang, J. F. (2014). The role of obesity in cardiomyopathy and nephropathy. Curr. Pharm. Des. 20, 1409-1417. doi: 10.2174/ 13816128113199990562

Walewski, J. L., Ge, F., Gagner, M., Inabnet, W. B., Pomp, A., Branch, A. D., et al. (2010). Adipocyte accumulation of long-chain fatty acids in obesity is multifactorial, resulting from increased fatty acid uptake and decreased activity of genes involved in fat utilization. Obes. Surg. 20, 93-107. doi: 10.1007/s11695009-0002-9

Wang, Q., Zhu, C., Sun, M., Maimaiti, R., Ford, S. P., Nathanielsz, P. W., et al. (2019). Maternal obesity impairs fetal cardiomyocyte contractile function in sheep. FASEB J. 33, 2587-2598. doi: 10.1096/fj.201800988R

Wang, S., and Ren, J. (2018). Obesity paradox in aging: from prevalence to pathophysiology. Prog. Cardiovasc. Dis. 61, 182-189. doi: 10.1016/j.pcad.2018. 07.011

Wang, Y., Liu, T., Liu, Y., Chen, J., Xin, B., Wu, M., et al. (2019). Coronary artery disease associated specific modules and feature genes revealed by integrative methods of WGCNA. Metade Mach. Learn. Gene 710, 122-130. doi: 10.1016/j. gene.2019.05.010

WHO (2000). Obesity: preventing and managing the global epidemic. Report of a WHO consultation. World Health Organ. Tech. Rep. Ser. 894, 1-253.

$\mathrm{Xu}, \mathrm{X} . \mathrm{H}$., and Ren, J. (2013). "Regulation of autophagy in obesity-induced cardiac dysfunction," in Autophagy: Caner, Other Pathologies, Inflammation, Immunity, and Infection, ed. M. A. Hayat (Cambridge, MA: Academic Press), 329-340.

Zhang, Y., and Ren, J. (2016). Epigenetics and obesity cardiomyopathy: From pathophysiology to prevention and management. Pharmacol. Ther. 161, 52-66. doi: 10.1016/j.pharmthera.2016.03.005

Zhang, Y. Y., and Ren, J. (2015). "Leptin and obesity," in Obesity - A practical Guide, ed. S. A. I. Ahmad (Berlin: Springer Press), 45-58.

Zhao, J., Lv, T., Quan, J., Zhao, W., Song, J., Li, Z., et al. (2018). Identification of target genes in cardiomyopathy with fibrosis and cardiac remodeling. J. Biomed. Sci. 25:63. doi: 10.1186/s12929-018-0459-8

Conflict of Interest: The authors declare that the research was conducted in the absence of any commercial or financial relationships that could be construed as a potential conflict of interest.

Copyright $\odot 2020$ Tao, Wang, Li, Zheng and Liang. This is an open-access article distributed under the terms of the Creative Commons Attribution License (CC BY). The use, distribution or reproduction in other forums is permitted, provided the original author(s) and the copyright owner(s) are credited and that the original publication in this journal is cited, in accordance with accepted academic practice. No use, distribution or reproduction is permitted which does not comply with these terms. 